\title{
Outcomes following resection of non-small cell lung cancer in octogenarians
}

\author{
Jaideep Vazirani*1 \\ Johanna Moraes \\ Stephen Barnett ${ }^{2,3,4,5}$ \\ Douglas F Johnson ${ }^{6,10}$ \\ Simon Knight ${ }^{3,5}$ \\ Alistair Miller $^{7}$ \\ Gavin Wright ${ }^{2,4,5,8}$ \\ Naveed Z Alam ${ }^{5,8}$ \\ Matthew Conron ${ }^{9,10}$ \\ Louis B Irving ${ }^{1,10,11}$ \\ Phillip Antippa $a^{2,4,5}$ \\ Daniel P Steinfort ${ }^{1,10,11}$
}

1. Department of Respiratory and Sleep Medicine, The Royal Melbourne Hospital

2. Department of Cardiothoracic Surgery, The Royal Melbourne Hospital

3. Department of Thoracic Surgery, Austin Hospital

4. Division of Cancer Surgery, Peter MacCallum Cancer Centre

5. Department of Surgery, University of Melbourne

6. Department of Medicine, Royal Melbourne Hospital

7. Department of Respiratory \& Sleep Medicine, Monash Health

8. Department of Cardiothoracic Surgery, St Vincent's Hospital

9. Department of Respiratory and Sleep Medicine, St Vincent's Hospital

10. Department of Medicine, University of Melbourne

11. Department of Cancer Medicine, Peter MacCallum Cancer Centre

Tables; 1

Figures; 1

Word count; 3491 (Abstract 250, Text 2799)

This is the author manuscript accepted for publication and has undergone full peer review but has not been through the copyediting, typesetting, pagination and proofreading process, which may lead to differences between this version and the Version of Record. Please cite this article as doi: $10.1111 /$ ans.14861

This article is protected by copyright. All rights reserved. 
Dr. Jaideep Vazirani

jaideepvazirani55@gmail.com

605/55 Queens Rd, Melbourne 3004

$+61433966650$

This article is protected by copyright. All rights reserved. 
Background

The treatment of choice for early stage Non-Small Cell Lung Cancer (NSCLC) is surgical resection. Little is known about the short and long-term outcomes amongst very elderly patients. We sought to determine predictors of short and long-term survival amongst octogenarians undergoing curativeintent resection for NSCLC in Victoria,Australia.

Methods

We retrospectively reviewed data from all patients aged $\geq 80$ who underwent curative-intent resection for NSCLC over 12 years (01/2005-12/2016) across five tertiary centres. We examined effect of age,stage of disease, extent of surgery and lung function on short and long-term survival.

Results

200 patients aged $\geq 80$ underwent curative-intent resections. Mortality at 30 and 120 days was $2.9 \%$ and $5.9 \%$,respectively. Increased early mortality was observed amongst those $\geq 83$ years old, at 30 days $(6.8 \%$ vs. $0.8 \%, p=0.044)$ and 120 days ( $12.2 \%$ vs. $2.3 \%, p=0.0096)$. Early mortality was highest among patients $\geq 83$ years requiring lobectomy, compared to sub-lobar resection at 120 days ( $17 \%$ vs $3.8 \%, p=0.019)$. Long term survival was predicted by age and stage of disease. Amongst patients with stage I disease aged $<83$ years, lobectomy was associated with superior 5-year survival, compared to sub-lobar resection ( $83 \%$ vs $61 \% \mathrm{p}=0.02)$.

Conclusion

In carefully selected elderly patients undergoing curative-intent resection of early stage NSCLC, both short and long-term outcomes appear consistent with younger historical cohorts. Early mortality was

This article is protected by copyright. All rights reserved. 
associated with lobectomy in those with advanced age. Older patients undergoing lobectomy appeared to be at highest risk for early mortality, while younger patients with stage I disease undergoing at least lobectomy appear to have the best long-term survival.

\section{Introduction}

Lung cancer is the leading cause of cancer-specific mortality worldwide (1). It is typically a disease of the elderly and its management in older patients is of growing concern, particularly in the context of an increasingly geriatric population (2). Although complete resection with lobectomy and mediastinal lymph node dissection or sampling remains the treatment of choice for early stage Nonsmall Cell Lung Cancer (NSCLC)(3) there is recent evidence to suggest that very elderly patients may be at significantly increased risk of peri-operative morbidity and mortality $(4,5)$. Currently published surgical outcomes in octogenarians vary considerably, with reported 5-year overall survival rates between $24-66 \%$ and peri-operative mortality rates ranging from $0-12.5 \%(5-7,21)$.

We present this cohort of octogenarians and nonagenarians who underwent curative-intent resection for NSCLC at five tertiary campuses in Melbourne, Australia.

This article is protected by copyright. All rights reserved. 


\section{Methods}

\section{Data sources}

We retrospectively examined prospectively-maintained single institution databases from five tertiary hospital campuses in Melbourne, Australia: The Royal Melbourne Hospital, St Vincent's Hospital, Peter MacCallum Cancer Centre, Monash Medical Centre and The Austin Hospital. These included co-located private hospital services at those campuses.

\section{Participants}

We identified all patients aged 80 years or older (at the time of operation), who underwent curativeintent resections of NSCLC between January 2005 and December 2016 at the aforementioned institutions. Patients with more than one primary tumour operated on at different time points were recorded as separate index cases. Patients with more than one primary tumour removed in one surgical episode were recorded as a single case.

\section{Data collection}

From the prospective databases, we recorded patient demographic, surgical and tumour factors. These included age, sex, lung function [forced expiratory volume in 1 second (FEV1), forced expiratory ratio (FER) and carbon monoxide diffusing capacity-lung (DLCO)], procedure type, pneumonectomy, bilobectomy, lobectomy or sub-lobar resection (i.e. segmentectomy, wedge resection), surgical access technique (i.e. video-assisted thoracoscopic (VATS) vs. open thoracotomy), Tumour, Node, Metastasis (TNM) stage (7th edition)(10), and histological type. 
Where pathological data was missing, operative pathology reports were examined directly and staged according to the seventh lung cancer TNM staging system. Lung function tests, where not available in the prospective databases, were retrieved from clinical information systems from the participating sites.

Vital status and, where relevant, date of death were determined based on review of institutional records, as well as submission of individual data to government based registries (Victorian Cancer Registry and data from the Registry of Births, Deaths and Marriages). Notification of cancer diagnoses and deaths to these databases is a statutory requirement. Where individuals were not identified in Registry databases, survival time was based on the most recent date of clinical review.

\section{Statistical analysis}

Categorical variables were presented as simple proportions and compared using Fisher's exact test. Age was used as a continuous variable and dichotomized at the median and mean values. Continuous variables were expressed as median or mean \pm standard deviation (SD), with the MannWhitney test used to compare unpaired non-parametric continuous values. Survival differences were determined by the Cox proportional hazards method. All reported confidence intervals are two-sided. A p-value of less than 0.05 was considered significant. All p-values reported excluded missing data. Survival analyses were performed using RStudio Version 1.0.143 for Intel Mac OS X 10.12.5.

\section{Comparison with Australian Life Tables}

Using the Australian Bureau of Statistics life tables for Australia 2013-2015, the resected cohort were matched to the general population for the same sex, year of birth and age at time of surgery. A rate 
table was generated with the expected annual death rate for the matching general population. The $R$ function 'survexp()' function was used to create a survival hazard which could be superimposed, using the R function 'lines()', onto a survival curve for the studied cohort.

This article is protected by copyright. All rights reserved. 


\section{Results}

\section{Demographics}

Two hundred patients were identified across the five sites, having 205 resections for NSCLC. The median and mean age of patients in our sample was 82 and 83 years at operation, respectively, and $20 \%$ of the cohort $(n=42)$ was $\geq 85$. Only two patients were aged over 90 years. Males comprised $63 \%$ of the cohort.

The majority of resections were lobectomies (59\%) followed by sub-lobar resection (39\%). Sub-lobar resections included 45 wedge resections and 34 anatomical segmentectomies (inclusive of lingulectomies and any segment-sparing lobectomies). Three patients underwent bilobectomy and three underwent pneumonectomy. Analyses of lobectomy outcomes was performed with and without pneumonectomies and bilobectomies, which were only performed in patients $<83$ years.

Most patients had pathological stage I disease (66\%), followed by stage II (22\%) and stage III (12\%). The most common histological type was adenocarcinoma (50\%) followed by squamous cell carcinoma (39\%) and other (11\%). The median FER of the sample was $70 \%( \pm 15.5)$, and median FEV1 was $89 \%( \pm 20)$. Amongst those requiring lobectomy, median FER and FEV1 were $70 \%$ and $92 \%$ respectively, as compared to $71 \%$ and $83 \%$ for those undergoing sub-lobar resection. FEV1 and FER values were not significantly different between patients undergoing sub-lobar resection and lobectomy ( $p=0.32$ and 0.62 , respectively). DLCO was likewise not significantly different between patients undergoing lobectomy and sub-lobar resection (data not presented).

\section{Early mortality}

Overall early mortality at 30 and 120 days was $2.9 \%$ (6/205), and 5.9\% (12/205) respectively.

This article is protected by copyright. All rights reserved. 
Early mortality was increased amongst those $\geq 83$ years old at both 30 days ( $6.8 \%$ vs. $0.8 \% ; p=0.044$ ) and 120 days ( 12.2 vs. $2.3 \%$; $p=0.0096$ ). Extent of resection (lobectomy vs sub-lobar) was nonsignificantly associated with increased mortality at 30 days ( $4.8 \%$ vs $0 \% ; p=0.13$ ). Early mortality appeared highest amongst those $\geq 83$ years requiring lobectomy, with $17 \%$ mortality at 120 days $(p=0.019)$.

Spirometry parameters and DLCO did not significantly impact early mortality (data not presented). Reduced lung function (FER $\leq 70$ ) was not associated with increased early mortality at 30 and 120 days ( $p=0.094$ and 0.467 respectively for lobectomy; $p=1.0$ and 0.290 respectively for sub-lobar resection).

\section{Long term survival}

Increased age and stage were independently associated with reduced 5-year survival ( $p=0.019$ and $\mathrm{p}=0.012$, respectively). Median survival was 8.1 years, 5.4 years and 2.4 years for stage I, II and III disease, respectively (figure 1a). The respective 5 -year survival rates were $60 \%, 49 \%$ and $20 \%$.

Extent of resection was not predictive of survival, regardless of whether pneumonectomies and bilobectomies were included with the lobectomy group or not ( $p=0.61$ and 0.50 , respectively). Of the patients with stage I NSCLC ( $n=135)$, neither extent of resection (lobectomy vs sub-lobar resection), nor spirometry parameters and DLCO (data not presented) were statistically linked to overall long term survival.

Amongst stage I patients, reduced 5-year survival was observed in patients aged $\geq 83$ (figure $1 \mathrm{~b}$ ), and age as a continuous variable was also associated with poorer survival in univariate analysis $(p=0.008)$. Univariate analysis for stage I patients showed no association between the following 
variables and overall survival: Campus, Sex, Extent of resection or Access technique (VATS versus open). As age was the only predictive variable in univariate analysis with a $p$-value $<0.10$, no multivariate analysis was performed.

Further analysis of stage I patients aged $<83$ years revealed superior long term survival amongst those undergoing lobectomy, as compared to sub-lobar resection (5 year survival $83 \%$ vs $61 \%$; $p=0.02$; figure $1 c)$. In this subgroup, there were no other variables predictive of outcome by univariate analysis, including age as a continuous variable. Multivariate analysis was performed using extent of resection and access technique (table 1), and this showed extent of resection persisting as the independent predictor $(p=0.045)$

\section{Comparison with Australian Life Tables}

The survival curve for the entire resected cohort was superimposed with the matched cohort from the Australian Bureau of Statistics 2013-2015 release. The curves completely overlapped until 7.5 years post resection (5). According to the life tables, an Australian female aged 85 years at the midpoint of this study in 2011, had a median survival of 4.33 years and an expected 5 -year survival probability of $45 \%$. (figure $1 d$ )

This article is protected by copyright. All rights reserved. 


\section{Discussion}

We have examined outcomes in five tertiary centres for carefully selected octogenarian patients with early stage NSCLC. We have observed excellent short term outcomes in patients undergoing sub-lobar resection and lobectomy that is consistent with previously published cohorts. Those at highest risk of early mortality were patients aged $\geq 83$ years requiring lobectomy, where 120 -day mortality was $17 \%$. Our findings regarding factors influencing short term and long term survival are otherwise consistent with previous studies $(5,11-13)$.

Interestingly, the best long term survival was achieved amongst octogenarians aged $<83$ with stage I disease who underwent lobectomy. The very choice of sub-lobar resection may relate to comorbidity, which then competes with cancer as a risk factor for death. Therefore the lower survival rate may just reflect selection bias.

Pathologic upstaging to Stage III disease was observed in $10 \%$ of patients, consistent with previous studies. We have only directly compared long-term outcomes among patients with Stage I NSCLC, as we did not have information regarding adjuvant therapy in patients with Stage II-III NSCLC, making interpretation of outcomes difficult in this group. Overall survival of Stage I NSCLC patients was superior for younger (<83 years) patients undergoing lobectomy, as compared to sub-lobar resection. Our findings provide significant reassurance that surgical management remains appropriate in carefully selected octogenarian patients with NSCLC. Concerningly, a recent large population based study from the UK that examined more than 10,000 cases of NSCLC ( $8 \%$ octogenarian, $n=860$ ), found the 90 -day mortality in all octogenarians to be close to $11 \%$, a figure much higher than that seen in our study and other smaller series (4). Consistent with our findings, this UK study found advanced age to be the most important predictor of early mortality, 
independent of other demographic, tumour and comorbid factors (4). Other population-based studies have found significantly worse outcomes than smaller series, possibly related to hospital volume and patient selection (14). This cohort, whilst not population-based, is representative of the vast majority of lung cancer resections in the State of Victoria.

A likely explanation for the lower mortality seen in our study and amongst other studies $(5,11-13)$, may include cautious patient selection. Our findings may indicate that selection processes in tertiary centres with dedicated thoracic oncology departments may be accurate in identification of patients at low risk of poor surgical outcomes, however this retrospective study is not able to elucidate any specific selection criteria to aid clinicians. We have not documented patient selection strategies at each centre, though our findings are reassuring that current assessment techniques are appropriate when selecting elderly patients for surgery. To optimize patient selection, a better understanding of risk factors for poor outcomes in the elderly is necessary, beyond the standard respiratory function tests.

Although we found that octogenarians have some increased surgical risk compared to historical controls(4) this increase does not seem great enough to preclude surgery for NSCLC amongst highly selected elderly patients. Increases in morbidity and mortality in octogenarians may be expected due to declining physiological reserve and increased incidence of comorbidity and disability in this population $(15,16)$. Today, Australian men and women aged 85 years can expect to live for a further 6.2 and 7.2 years respectively which is more than 2 years greater than in the early $1990 \mathrm{~s}(17,18)$. Consistent with this significantly increased life expectancy, lung cancer remains the most common cause of death in elderly patients who develop this diagnosis. Furthermore, it is important to consider the poor prognosis for patients with NSCLC that do not undergo any treatment. Estimated 
5-year survival in patients with stage I NSCLC, who do not receive any form of treatment, has been shown to be as low as $7 \%(19)$, suggesting any treatment is beneficial even in very elderly patients $(12,20)$. We have demonstrated that the survival probability of the resected cohort is the same as the general population matched for age and sex at the time of surgery. Our interpretation of this somewhat surprising finding is that the individuals selected for surgery are fitter than the average matched population, and then surgery removes the medium to long term risk of cancer death.

Finally we found that those $\geq 83$ years had worse short and long term survival outcomes. The median age of octogenarians in our sample was 82.6 years, suggesting that it was primarily younger, and therefore potentially healthier, octogenarians that undergo surgery. Few studies have looked at whether there are significantly different outcomes in younger versus older octogenarians, but age over 85 years has previously been associated with increased 90-day mortality (4). Further research in this area is necessary to establish if this association holds true, as it might be that acceptable early mortality outcomes are only found in a younger subset of octogenarians.

Patient selection is critical in this patient group, and future studies are required to examine specific selection processes to optimize therapeutic outcomes, especially with the advent of effective noninvasive therapies such as stereotactic ablative body radiotherapy (SABR). However, the very patients that require lobectomy may be anatomically unsuitable for SABR and require conventional fractionated radiotherapy over 6 weeks, with its intendant increased morbidity (8-9).

We are unable to determine the rate of 'elective' versus 'compromise' sub-lobar resection in the $\geq 83$ year group. However, given the more favourable outcome, it is possible that when anatomical factors and stage allow, sub-lobar resection should be considered the gold standard procedure in this group.

This article is protected by copyright. All rights reserved. 


\section{Limitations}

As already indicated our study had a number of limitations, in particular missing and unrecorded data as a result of the retrospective nature of the study and some data being unavailable in deidentified databases. It is possible that for some variables our sample sizes might have been too small to determine statistically significant results. However our main finding regarding significantly elevated mortality in older subsets of octogenarians requiring extended resection were not compromised and therefore our findings provide important guidance to clinicians undertaking care of this subgroup. Future prospective studies are required to confirm our findings and to more accurately examine variables that may influence peri-operative morbidity and mortality.

An advantage of our study over many previous case series was our multi-centre approach. Patients from multiple Melbourne hospitals were included in our sample, thereby giving a more representative view of the local octogenarian population.

We do not have information regarding patients who were considered ineligible for resection of early stage NSCLC, specifically their performance status or co-morbidities. As patients were identified based on the retrospective pathological diagnosis of NSCLC, we were unable to assess the morbidity and mortality for patients submitted for surgery on the suspicion of NSCLC. A prospective study including this group would be valuable. There is likely a selection bias towards medically fitter patients, which we are unable to elucidate.

This article is protected by copyright. All rights reserved. 


\section{Conclusion}

In this highly selected group of octogenarian patients, short and long term outcomes in patient subsets following surgery were consistent with outcomes in younger cohorts, suggesting advanced age itself should not be a contraindication to curative-intent surgery amongst octogenarians with NSCLC. Older octogenarians undergoing lobectomy experienced high rates of 120-day mortality. More research into the effect of comorbidities and performance status on perioperative mortality in this subgroup is needed to select only the lowest operative risk patients for surgery. Younger octogenarians with stage I NSCLC undergoing lobectomy appear to have the best long term survival, comparable to that reported for much younger cohorts.

Surgical resection remains an appropriate treatment for early stage NSCLC in carefully selected octogenarian patients. Further research into risk factors for poor outcomes in the very elderly is necessary to guide selection of appropriate octogenarian patients and to minimize peri-operative morbidity and mortality among this group.

This article is protected by copyright. All rights reserved. 


\section{REFERENCES}

1. Mokhles S, Nuyttens JJ, Maat AP, Birim O, Aerts JG, Bogers AJ, et al. Survival and Treatment of Non-small Cell Lung Cancer Stage I-II Treated Surgically or with Stereotactic Body Radiotherapy: Patient and Tumor-Specific Factors Affect the Prognosis. Ann Surg Oncol. 2015;22(1):316-23.

2. Gonzalez-Aragoneses F, Moreno-Mata N, Simon-Adiego C, Penalver-Pascual R, Gonzalez-Casaurran G, Perea LA. Lung cancer surgery in the elderly. Critical reviews in oncology/hematology. 2009;71(3):266-71.

3. Howington JA, Blum MG, Chang AC, Balekian AA, Murthy SC. Treatment of stage I and II non-small cell lung cancer: Diagnosis and management of lung cancer, 3rd ed: American College of Chest Physicians evidence-based clinical practice guidelines. Chest. 2013;143(5 Suppl):e278S-313S.

4. Powell HA, Tata LJ, Baldwin DR, Stanley RA, Khakwani A, Hubbard RB. Early mortality after surgical resection for lung cancer: an analysis of the English National Lung cancer audit. Thorax. 2013;68(9):826-34.

5. Guerra M, Neves P, Miranda J. Surgical treatment of non-small-cell lung cancer in octogenarians. Interactive cardiovascular and thoracic surgery. 2013;16(5):673-80.

6. Okami JH, M; Asamura, H.; Goya, T.; Koshiishi, Y.; Sohara, Y.; Eguchi, K .; Mori, M.; Nakanishi, Y.; Tsuchiya, R.; Miyaoka, E. Pulmonary Resection in Patients Aged 80 years or over with Clinical stage I Non-small cell Lung cancer 
Prognostic factors for Overall Survival and Risk factors for Post-operative complications. J Thorac Oncol. 2009;4(10):1247-53.

7. Hope WB, W.; Stephenson, J.; Kalbaugh, C.; Blackhurst, D.; Taylor, S. Lung cancer resection in octogenaians: A reasonable approach for our aging population. The American Surgeon. 2007;73:22-4.

8. Ebright MI, Fernando HC. Surgical resection or stereotactic body radiation therapy in elderly patients with early-stage lung cancer: evolving treatment algorithms and a call for reliable comparisons. Seminars in thoracic and cardiovascular surgery. $2011 ; 23(2): 93-5$

9. Solda F, Lodge M, Ashley S, Whitington A, Goldstraw P, Brada M. Stereotactic radiotherapy (SABR) for the treatment of primary non-small cell lung cancer; systematic review and comparison with a surgical cohort. Radiotherapy and oncology : journal of the European Society for Therapeutic Radiology and Oncology. 2013;109(1):1-7.

10. Goldstraw P, Crowley J, Chansky M, Giroux D, Groome P, Rami-Porta R, et al. The IASLC Lung Cancer Staging Project: Proposals for the Revision of the TNM Staging Groupings in the Forthcoming (Seventh) Edition of the TNM Classification of Malignant Tumours. Journal of thoracic oncology. 2007;2(8):706-14.

11. Fanucchi O, Ambrogi MC, Dini P, Lucchi M, Melfi F, Davini F, et al. Surgical treatment of non-small cell lung cancer in octogenarians. Interactive cardiovascular and thoracic surgery. 2011;12(5):749-53.

This article is protected by copyright. All rights reserved. 
12. Dell'Amore A, Monteverde M, Martucci N, Sanna S, Caroli G, Stella F, et al. Early and long-term results of pulmonary resection for non-small-cell lung cancer in patients over 75 years of age: a multi-institutional study. Interactive cardiovascular and thoracic surgery. 2013;16(3):250-6.

13. Saha SP, Bender M, Ferraris VA, Davenport DL. Surgical treatment of lung cancer in octogenarians. Southern medical journal. 2013;106(6):356-61.

14. Finlayson E, Fan Z, Birkmeyer JD. Outcomes in octogenarians undergoing highrisk cancer operation: a national study. J Am Coll Surg. 2007;205(6):729-34.

15. Endoh H, Yamamoto R, Satoh Y, Kuwano H, Nishizawa N. Risk analysis for pulmonary resection for elderly patients with lung cancer. Surgery Today. 2013;43:51416. Janssen-Heijnen M, Maas H, Houterman S, Lemmens V, Rutten H, Coeberg J. Comorbidity in older surgical cancer patiens: influence on patient care and outome. European Journal of Cancer. 2007;43:2179-93.

17. AIHW. Older Australians at a glance. 4 ed. Canberra: Australian Institute of Health and Welfare; 2007.

18. Australian Bureau of Statistics. Life Tables, States, Territories and Australia, 20132015. Catalogue: 3302.0.55.001. http://www.abs.gov.au/ausstats/wmdata.nsf/activeimages/xlbutton/\$File/button_xls_ 3.png

19. Raz DJ, Zell JA, Ou SH, Gandara DR, Anton-Culver H, Jablons DM. Natural history of stage I non-small cell lung cancer: implications for early detection. Chest. 2007;132(1):193-9. 
20. Varlotto JM, Decamp MM, Flickinger JC, Lake J, Recht A, Belani CP, et al. Would screening for lung cancer benefit 75- to 84-year-old residents of the United States? Frontiers in oncology. 2014;4:37.

21. Voltolini L, Rapicetta C, Ligabue T, Luzzi L, Scala V, Gotti G. Short- and long-term results of lung resection for cancer in octogenarians. Asian cardiovascular \& thoracic annals. 2009;17(2):147-52. 


\section{University Library}

\section{- M M N E R VA A gateway to Melbourne's research publications}

Minerva Access is the Institutional Repository of The University of Melbourne

\section{Author/s:}

Vazirani, J;Moraes, J;Barnett, S;Johnson, DF;Knight, S;Miller, A;Wright, G;Alam, NZ;Conron, M;Irving, LB;Antippa, P;Steinfort, DP

Title:

Outcomes following resection of non-small cell lung cancer in octogenarians

Date:

2018-12-01

\section{Citation:}

Vazirani, J., Moraes, J., Barnett, S., Johnson, D. F., Knight, S., Miller, A., Wright, G., Alam, N. Z., Conron, M., Irving, L. B., Antippa, P. \& Steinfort, D. P. (2018). Outcomes following resection of non-small cell lung cancer in octogenarians. ANZ JOURNAL OF SURGERY, 88 (12), pp.1322-1327. https://doi.org/10.1111/ans.14861.

Persistent Link:

http://hdl.handle.net/11343/284559 